\title{
Évaluation de la potabilité chimique des eaux souterraines dans un bassin versant tropical : cas du Sud-Ouest de la Côte d'Ivoire
}

\author{
T.K. YAO ${ }^{1 *}$, M-S. OGA ${ }^{1}$, O. FOUCHÉ $^{2}$, D. BAKA ${ }^{1}$, C. PERNELLE $^{3}$ et J. BIEMI ${ }^{1}$ \\ ${ }^{1}$ Université de Cocody Abidjan, UFR-STRM, 22 BP 582 Abidjan 22, Côte d'Ivoire. \\ ${ }^{2}$ Conservatoire National des Arts et Métiers de Paris, Chaire de Géotechnique, 2 rue Conté, 75003 Paris, \\ France. \\ ${ }^{3}$ Conservatoire National des Arts et Métiers de Paris, Chaire de Chimie Analytique, 2 rue Saint Martin, 75141 \\ Paris cedex 03, France. \\ *Auteur correspondant, E-mail : koffiyao@ymail.com, Tel. : +22505000107
}

\section{RESUME}

L'étude propose un état des lieux de la qualité chimique des eaux souterraines ainsi que leur situation vis-à-vis d'éléments toxiques d'origine anthropique (nitrate et pesticides) dans la nouvelle boucle du cacao (Sud-Ouest de la Côte d'Ivoire). Cinquante-six échantillons d'eau souterraine et de surface ont été prélevés au cours de deux campagnes de terrain puis analysés en se basant sur seize paramètres physico-chimiques. Les résultats montrent que les eaux souterraines ont pour la majorité un $\mathrm{pH}$ neutre. Elles sont à $34 \%$ très faiblement minéralisées; seulement $21 \%$ présente une conductivité supérieure à $500 \mu \mathrm{S}_{\mathrm{S}} \mathrm{cm}^{-1}$. Ces eaux sont pour la plupart bicarbonatées dont une moitié est alcaline et l'autre calco-magnésienne. La qualité chimique est satisfaisante pour $80 \%$ des eaux étudiées au regard des normes OMS. Certaines ont même des teneurs voisines à certaines eaux minérales de commerce. En revanche, d'autres présentent des teneurs très critiques en nitrate, en sulfate et en fer. Les fortes teneurs en nitrate proviennent des activités humaines démontrant que les ressources en eau de la région ne sont pas à l'abri d'une pollution par les pesticides qui sont fortement utilisés dans les plantations de cacao, café, hévéa et palmier à huile ainsi que dans les maraîchers.

(c) 2012 International Formulae Group. All rights reserved.

Mots clés: Qualité physico-chimique, pesticides, nitrate, hydrochimie, Côte d’Ivoire.

\section{INTRODUCTION}

Les eaux issues des nappes souterraines et superficielles sont des ressources exploitées par l'homme pour divers usages (Margat, 2008). La composition chimique d'une eau issue du milieu naturel est très variable. Elle dépend de la nature géologique du sol d'où elle provient et aussi des substances réactives qu'elle aurait pu rencontrer lors de l'écoulement. Ainsi, la composition quantitative et qualitative de l'eau souterraine en matières en suspension et dissoutes, de nature minérale ou organique, détermine sa qualité (Jain et al., 2005). Cependant, cette qualité peut être altérée lorsque des substances extérieures entrent en contact avec la nappe aquifère. Tel est le cas des substances indésirables et toxiques qui rendent l'eau souterraine impropre pour la consommation domestique (Traoré et al., 2006). L'utilisation intensive des ressources naturelles et l'accroissement des activités humaines ont 
engendré de graves problèmes sur la qualité des eaux souterraines. Dans les pays en développement, obtenir de l'eau saine pour la consommation humaine est devenu un sérieux problème du fait d'un manque de protection de l'environnement (Ayraud, 2005). Par conséquent, l'eau qui est source de vie, devient source de maladies. Dans le SudOuest ivoirien, l'approvisionnement en eau potable des populations, surtout rurales est assuré parfois par des forages. La qualité chimique et microbiologique de cette eau n'a pas fait l'objet d'étude. En effet, lors de la mise en service quelques analyses d'ions sont faites. Mais, celles-ci ne sont pas toujours complètes pour apprécier véritablement leur qualité chimique. Il convient pour le besoin de santé publique de cerner la qualité chimique des eaux souterraines de cette partie de la Côte d'Ivoire. La présente étude se propose, d'une part, de caractériser et de montrer la spécificité chimique des eaux souterraines de Soubré et, d'autre part, de faire l'inventaire des causes de pollution d'origine naturelle et anthropique et de souligner l'impact que pourrait avoir cette pollution sur la qualité des eaux souterraines de la zone d'étude sur la base des fortes teneurs de nitrates. Elle se structure autour de trois grandes parties qui abordent respectivement le matériel et la méthodologie mis en œuvre, les principaux résultats issus de l'analyse et le traitement des données chimiques et enfin une discussion qui met en relations nos résultats et ceux des travaux en hydrochimie en zone de socle cristallophyllien et métamorphique du Ghana et de la Côte d'Ivoire. Dans cette partie, nous comparons la composition chimique de certaines eaux étudiées avec les eaux minérales de commerce et présentons enfin, les conséquences de la pollution au nitrate et son corollaire.

\section{MATÉRIEL ET MÉTHODES \\ Cadre d'étude}

Le domaine d'étude d'une superficie d'environ $8590 \mathrm{~km}^{2}$, est localisé au SudOuest de la Côte d'Ivoire entre les latitudes $5^{\circ} 19$ et $6^{\circ} 34$ et les longitudes $6^{\circ} 12$ et $7^{\circ} 08$
(Figure 1). Du point de vue géomorphologique, il fait partie d'une vaste pénéplaine légèrement ondulée, d'altitude moyenne égale à $200 \mathrm{~m}$ et inclinée vers la mer au sud avec une pente moyenne de $1 \%$ o (Tahoux, 1993). Cette région est drainée par le fleuve Sassandra et bénéficie d'un climat subéquatorial (chaud et humide). Les pluies y sont abondantes (entre $1600 \mathrm{~mm}$ et 1800 $\mathrm{mm}$ ) et atteignent un maximum vers juin et juillet. Les températures oscillent entre $26{ }^{\circ} \mathrm{C}$ et $32{ }^{\circ} \mathrm{C}$ au cours de l'année (Yao, 2009). L'approvisionnement en eau potable de la population se fait généralement aux moyens de puits et forages équipés de pompes à motricité humaine ou électrique dont les profondeurs varient entre $10 \mathrm{~m}$ et $120 \mathrm{~m}$ (Yao, 2009). Ces ouvrages hydrauliques captent les eaux des aquifères de fissures des formations géologiques appartenant au domaine éburnéen (2 500 à $1550 \mathrm{Ma}$ ). Il y affleure de façon très discontinue des granitoïdes intrusifs dans un encaissant métamorphique (gneiss et migmatites) plus ancien (Figure 2). Ces massifs apparaissent comme des satellites d'une coupole d'anatexie constituée d'un noyau granodioritique entouré de migmatite, elle-même entourée d'une auréole de gneiss. On rencontre aussi dans la zone ouest de la région des roches flyschoïdes constituées de schistes à chlorite et séricite, de schistes gréseux, de schistes quartzeux et de grès feldspathiques tuffacés. Ces formations se sont différenciées du manteau terrestre vers 2200 à $2300 \mathrm{Ma}$ et caractérisent le début de l'époque du Birimien qui regroupe l'ensemble des formations de l'Afrique de l'Ouest et représentent la majeure partie du bouclier ouest africain (Papon et Lemarchand, 1973). Plusieurs phases de déformation ont affecté cet ensemble géologique ce qui a abouti à la mise en place d'une fracturation intense. Les fractures s'orientent majoritairement selon trois directions principales : SSO-NNE, SO$\mathrm{NE}$ et N-S. Quelques failles secondaires de longueur maximale $15 \mathrm{~km}$ et orientées NO-SE ou parfois $\mathrm{N}-\mathrm{S}$, marquées par des mylonites, se dispersent dans les granodiorites et les migmatites. 


\section{Échantillonnage et analyses physico- chimiques}

Pour la détermination des paramètres physico-chimiques, deux campagnes d'échantillonnage de l'eau souterraine et de surface ont été réalisées en mai 2007 et Mars 2008 suivant le protocole préconisé par Barcelona et al. (1985), Afnor (1997) et Thierrin et al. (2001). Au total, cinquante-six stations de prélèvement d'eau réparties sur le territoire comprenant cinquante-trois points d'eau souterraine et trois d'eau de surface (fleuve Sassandra ou ses tributaires) ont été choisies (Figure 2). Les échantillons d'eau souterraine ont été prélevés à partir des forages, directement de la pompe et en amont de tout système de traitement. Préalablement à l'échantillonnage, chaque forage a été purgée jusqu'à l'obtention d'une lecture stabilisée de $\mathrm{pH}$ et de conductivité électrique à l'aide de sondes portables (Ciba Corning et WTW LF 318). La dureté totale de l'eau (THt) a été déterminée par titrage volumétrique au moyen d'un coffret de test de dureté totale Aquamerck® contenant une solution de titrage et un réactif indicateur coloré (Noir Eriochrome T). L'alcalinité des eaux est déterminée sur le terrain à l'aide d'une languette de titre alcalimétrique (TA). Les méthodes d'analyses sont celles préconisées par les normes Afnor (1997) et par Rodier (1996). Avant le remplissage des bouteilles, celles-ci ont été lavées trois fois avec l'eau à prélever. Le remplissage des bouteilles a été fait à ras bord puis le bouchon vissé afin d'éviter tout échange gazeux avec l'atmosphère. L'eau prélevée a été filtrée au moment de l'échantillonnage à l'aide des filtres Millipores de $45 \mu \mathrm{m}$. Les échantillons destinés au dosage du nitrate ont été acidifiés à l'acide sulfurique $2 \mathrm{~N}$ et ceux destinés à l'analyse des cations ont été acidifiés à l'acide nitrique $16 \mathrm{~N}$. Tous les échantillons ont été conservés à $4 \quad{ }^{\circ} \mathrm{C}$ jusqu'à l'analyse en laboratoire. Les travaux de dosage ont lieu au laboratoire de la Chaire Génie Analytique du Conservatoire National des Arts et Métiers de Paris. Les teneurs en nitrate et en sulfate ont été déterminées par colorimétrie (spectrophotométrie) tandis que celles en bicarbonate et en chlorure ont été obtenues par titration. Les échantillons d'eau ont été analysés pour leurs teneurs en cations $(\mathrm{Ca}$, $\mathrm{Mg}, \mathrm{Na}, \mathrm{K}$,) par spectrométrie d'absorption atomique (AAS) et en éléments en trace (Fe, $\mathrm{Mn}, \mathrm{Al})$, par spectrométrie d'émission atomique par couplage inductif (ICP-AES). Le contrôle de la validité des résultats des analyses a été fait par le calcul des balances ioniques. L'analyse est de bonne qualité si la balance est inférieure ou égale à $5 \%$. Toutefois, pour les eaux de socle, où la minéralisation des eaux est souvent faible, une balance de $10 \%$ est acceptable (Hem, 1989). La recherche de la particularité des eaux souterraines du département de Soubré, nous a amené à comparer certaines eaux de socle de formations plutoniques et métamorphiques en Côte d'Ivoire et au Ghana. Les résultats des analyses physico-chimiques ont été également comparés aux valeurs guides de l'Organisation Mondiale de la Santé (OMS, 2008) et à la composition chimique de certaines eaux minérales. À défaut d'une étude complète sur le nitrate dans les eaux de Soubré, nous avons cherché à la lumière de certains travaux l'origine de l'abondance du nitrate dans les eaux souterraines et leur lien avec la pollution par les produits phytosanitaires. En effet, pour l'entretien des vergers de cacao et de café ou pour le désherbage des vallées pour la riziculture, les paysans ont recours à divers produits chimiques. Ceux qui ont été recensés sont connus sous leurs noms commerciaux : Actara, Tropicao, Basusine, Roundup etc. Ces produits, largement utilisés sans aucune attention présentent des effets très nocifs pour l'homme. C'est le cas du Roundup (herbicide non-sélectif) qui est utilisé en épandage. Il contient plusieurs substances toxiques notamment la molécule «active»: le glyphosate ((N-(phosphonométhyl)glycine, $\mathrm{C}_{3} \mathrm{H}_{8} \mathrm{NO}_{5} \mathrm{P}$ ), et des substances dites « inertes » que sont le POEA (adjuvant), AMPA et l'isopropylamine (Fanz et al., 1997). 


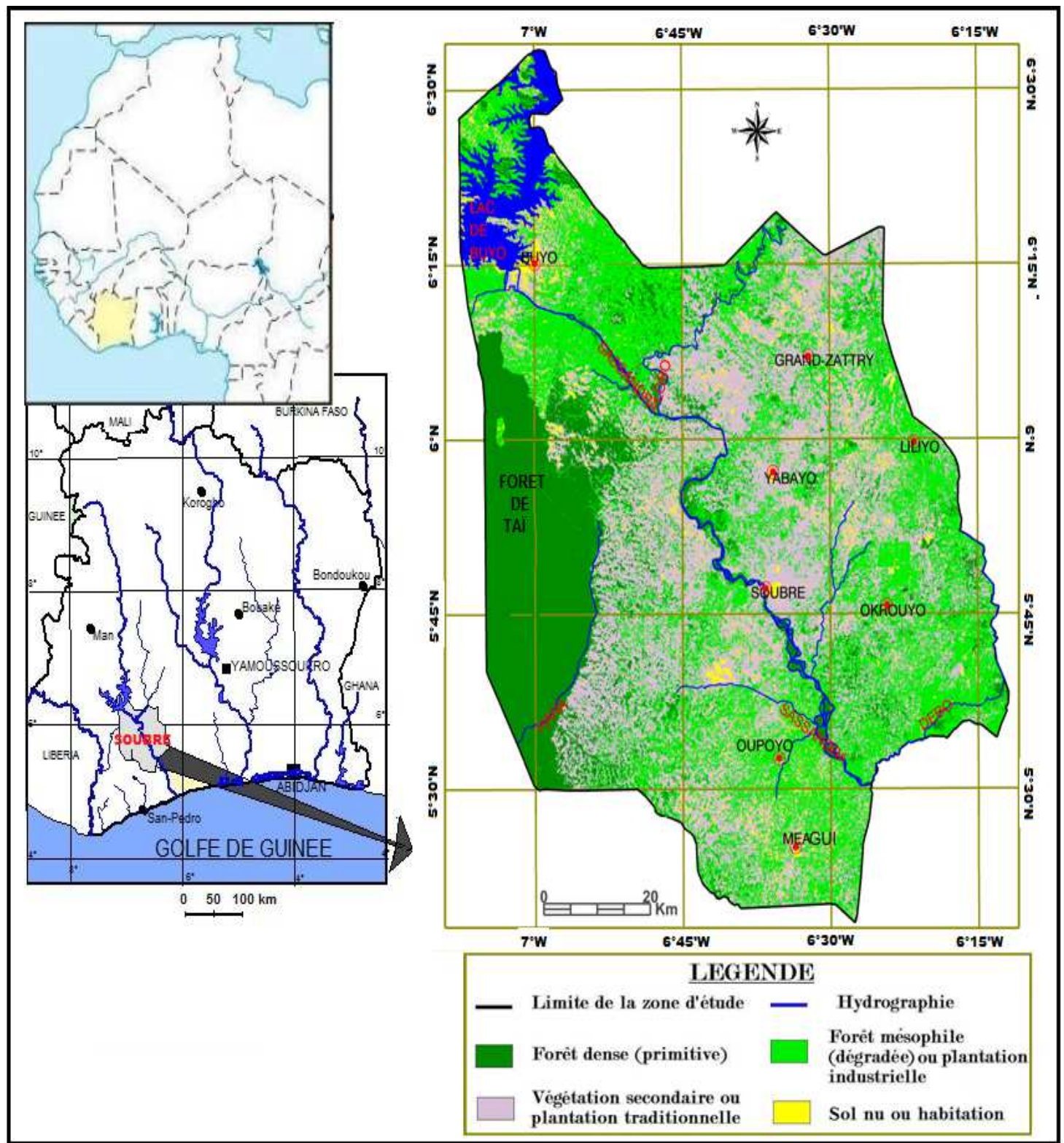

Figure 1 : Présentation de la zone d'étude.

\section{RÉSULTATS}

\section{Caractéristiques hydrochimiques des eaux}

Le Tableau 1 présente le résumé des résultats des analyses physico-chimiques des échantillons d'eau de cette étude. Dans l'ensemble, les résultats sont acceptables car $71 \%$ des échantillons des eaux analysées ont une balance ionique inférieure ou égale à $5 \%$. Celles qui présentent une balance ionique en déséquilibre restent dans une limite acceptable (entre $-8 \%$ et $+8 \%$ ). Elles indiquent pour la plupart des conductivités électriques supérieure à $400 \mu \mathrm{S} . \mathrm{cm}^{-1}$ (Figure 3). Les valeurs de température des eaux sont de 
l'ordre de $27 \pm 2$ et les $\mathrm{pH}$ sont voisins de la neutralité. Leurs teneurs en Matières Totales Dissoutes (MTD) sont relativement faibles, comprises entre $82 \mathrm{mg} . \mathrm{L}^{-1}$ (L37) et $732 \mathrm{mg} . \mathrm{L}^{-1}$ (L8) avec des valeurs extrêmes de $762 \mathrm{mg} . \mathrm{L}^{-1}$ et $1045 \mathrm{mg} . \mathrm{L}^{-1}$ observées respectivement dans les eaux de L39 et de M15. De même, les conductivités électriques (CE) oscillent entre $61 \mu \mathrm{S} . \mathrm{cm}^{-1}$ et $740 \mu \mathrm{S} . \mathrm{cm}^{-1}$ avec de fortes conductivités dans les eaux de L39 et M15 $\left(971 \mu \mathrm{S} . c m^{-1}\right.$ et $\left.1128 \mu \mathrm{S} . \mathrm{cm}^{-1}\right)$. Les conductivités peuvent être regroupées en 3 intervalles. $34 \%$ ont une conductivité électrique inférieure à $250 \mu \mathrm{S} . \mathrm{cm}^{-1}$, donc très faiblement minéralisées. Les $66 \%$ restant se répartissent en $45 \%$ d'eaux présentant une $\mathrm{CE}$ entre 250 et $450 \mu \mathrm{S} . \mathrm{cm}^{-1}$ et $21 \%$ indiquent une $\mathrm{CE}$ supérieure à $500 \mu \mathrm{S} . \mathrm{cm}^{-1}$. Le titre alcalimétrique complet (TAC) dans les échantillons d'eaux analysées était essentiellement dû aux ions bicarbonate. Le TAC se situait dans l'intervalle 1,4 à $34,7^{\circ} \mathrm{F}$, soit 5 à $85 \mathrm{mg} \cdot \mathrm{L}^{-1}$ de $\mathrm{CaCO}_{3}$. Cependant, près de $81 \%$ des eaux présente un TAC inférieur à $20{ }^{\circ} \mathrm{F}$ ce qui se traduit par de très faibles concentrations en ions bicarbonates. Sur le plan chimique, les eaux échantillonnées sont caractérisées par une très faible quantité d'ions potassium soit $9 \%$ des espèces cationiques et $4 \%$ de la totalité des ions analysés. Les teneurs vont de 0,1 meq. $\mathrm{L}^{-1}$ à 0,8 meq. $\mathrm{L}^{-1}$. Quant aux ions sodium, ils représentent en moyenne $31 \%$ des espèces cationiques. Le calcium et le magnésium représentent respectivement en moyenne $35 \%$ et $25 \%$ des cations. Ainsi, les alcalino-terreux dominent $\left(\mathrm{Ca}^{2+}+\mathrm{Mg}^{2+}\right)$ dans les eaux de la région de Soubré. Concernant les anions, les bicarbonates, avec $53 \%$ des anions représentent les anions les plus abondants. Ils ont des teneurs comprises entre 0,3 meq. $\mathrm{L}^{-1}$ (L44) et 6,9 meq. $\mathrm{L}^{-1}$ (L39). Les sulfates représentent $24 \%$ des anions avec des valeurs importantes dans certaines eaux souterraines, notamment dans celles de Néagreadji (9 meq. $\left.\mathrm{L}^{-1}\right), \quad$ Grand-Zattry $\quad\left(8,6\right.$ meq. $\left.\mathrm{L}^{-1}\right) \quad$ et Degaullekro (2,4 meq. $\left.\mathrm{L}^{-1}\right)$. Quant aux chlorures, ils représentent $15 \%$ des anions avec des teneurs comprises entre 0,09 meq. $\mathrm{L}^{-1}$ et 1,89 meq. $\mathrm{L}^{-1}$. Les nitrates ne représentent en moyenne que $6 \%$ des anions. Les teneurs sont comprises entre 0 et 1,6 meq. $\mathrm{L}^{-1}$. Certaines eaux indiquent des teneurs très élevées dont les remarquables sont Abokouamekro (1,6 meq. $\left.\mathrm{L}^{-1}\right)$ et à Sao (1,2 meq. $\left.\mathrm{L}^{-1}\right)$. Aussi, note-t-on que $18 \%$ des eaux ont des concentrations en nitrate qui excèdent la norme OMS (50 mg. $\mathrm{L}^{-1}$, soit 0,86 meq. $\mathrm{L}^{-1}$ ). Ce sont, dans l'ordre décroissant, L13, SAO, L14, L11, L28, L2, et L23. Dans l'ensemble, plus de $32 \%$ des eaux présentent des teneurs supérieures à $20 \mathrm{mg} \cdot \mathrm{L}^{-1}\left(0,3 \mathrm{meq} \cdot \mathrm{L}^{-1}\right)$. Les teneurs en fer représentent près de $80 \%$ des éléments en trace dosés. Celles du manganèse et de l'aluminium ne représentent respectivement que $14 \%$ et $6 \%$. Les teneurs en fer des eaux vont de 0 à $1,35 \mathrm{mg} . \mathrm{L}^{-1}$ alors que celles du manganèse oscillent entre 0 et 0,3 mg. $\mathrm{L}^{-1}$.

\section{Faciès hydrochimiques}

L'interprétation des valeurs des différents paramètres physico-chimiques précédents montrent que les souterraines de Soubré, sont majoritairement bicarbonatées (72\%), dont $50 \%$ des échantillons sont sodipotassiques et l'autre moitié sont calcomagnésiens. À l'intérieur d'un faciès hydrochimique, selon le cation qui vient en seconde position dans l'ordre croissant d'abondance, plusieurs variantes sont observées. Ainsi, au sein des eaux bicarbonatées calciques, on peut distinguer des eaux bicarbonatées calciques et magnésiennes (M6, M3, L32, etc.) et des eaux bicarbonatées calciques et sodiques (M2, M8, L31, etc.). Les eaux sulfatées (18\%) sont à dominance sodi-potassique. Le faciès chloruré est rencontré dans $10 \%$ des échantillons. Les eaux calco-magnésiennes ont des teneurs plus 
élévées en bicarbonate que les eaux alcalines. Les échantillons L28 et L44 ainsi que L13 sont des eaux à forte teneur en nitrate et en alcalins. Ils ont été classés dans le faciès correspondant à leur couple anion / cation le plus significatif après le nitrate. Les eaux calco-magnésiennes sont plus sulfatées que chlorurées alors que les eaux alcalines sont plus chlorurées que sulfatées. La Figure 4 montre que le faciès des eaux souterraines n'est pas lié à une formation géologique. Les faciès bicarbonatés calciques et bicarbonatés sodi-potassiques, qui sont les faciès dominants s'observent dans toutes les lithologies. Ce constat est valable aussi pour les autres faciès.

\section{Comparaison des eaux souterraines de Soubré avec certaines eaux de socle}

Les teneurs des éléments en trace, dont celles du fer sont présentées dans le Tableau 2 tandis que le Tableau 3 compare les éléments majeurs des eaux étudiées avec celles issues des travaux de Biémi (1992), Tapsoba (1995), Soro (2002), et Goné et al. (2005).

\section{Analyse comparée entre souterraine de Soubré et la directive de l'OMS}

La comparaison des mesures des éléments physico-chimiques (Tableau 4) avec les normes de l'OMS (2008) usuelles permet de faire certaines remarques. Dans l'ensemble, la quasi-totalité des eaux étudiées présentent des teneurs inférieures aux normes admissibles. À l'exception du nitrate, du calcium magnésium et du sulfate. La teneur maximale en sulfate presqu'identique à la norme admise. Les eaux en gras dans le Tableau 5 sont des eaux dites "spéciales" car elles présentent au moins deux paramètres chimiques ayant des teneurs hors norme. C'est le cas de l'eau de (M15 ; Négrea dji) qui indique des valeurs très élevées en sulfates et en calcium. Elle est donc spéciale car ayant des teneurs en ions qui surpassent celles de plusieurs eaux minérales (Tableau 6). D'ailleurs, l'eau de ce forage est dure (THT > 30 i.e $=60,4^{\circ} \mathrm{F}$, mousse difficilement) et n' est pas appréciée pas la population locale. Le Tableau 6 présente également les teneurs en ions des eaux spéciales : il s'agit des échantillons L39 et L8. Ils sont riches en fer, manganèse, magnésium, potassium et en sulfates pour L39.

\section{Cas du nitrate}

La Figure 5 et le Tableau 5 montrent une abondance de nitrate dans plusieurs échantillons. D'ailleurs, par endroits, sa teneur est supérieure à la norme admissible par l'OMS. Les fortes teneurs en nitrate sont préférentiellement observées dans les eaux à dominance alcaline et les groupes d'eau à faciès intermédiaires ou de mélange. Ce sont des eaux riches en $\mathrm{Na}$ et en $\mathrm{Ca}$. À priori, ce sont des eaux d'altérites qui circulent difficilement mais reçoivent du nitrate par infiltration. Cependant, quelques eaux de d'autres pôles sont riches en nitrate. Ce constat soulève le problème de l'origine de cet ion, en particulier pour les eaux des nappes de fissures qui sont censées être à l'abri d'une pollution directe car elles sont protégées par le régolite. À défaut, d'une étude sur l'origine de cet ion, nous avons à travers certains travaux montré que sa présence dans l'eau souterraine est une indication de la vulnérabilité des nappes de la région. 
T.K. YAO et al. / Int. J. Biol. Chem. Sci. 6(6): 7069-7086, 2012

Tableau 1: Caractéristiques physico-chimiques moyennes des eaux échantillonnées.

\begin{tabular}{|c|c|c|c|c|c|c|c|c|c|c|c|c|c|c|c|c|c|}
\hline \multirow{3}{*}{$\begin{array}{l}\text { Paramètres } \\
\text { statistiques }\end{array}$} & \multicolumn{6}{|c|}{ Analyses physico-chimiques } & \multicolumn{11}{|c|}{ Analyses chimiques } \\
\hline & \multicolumn{6}{|c|}{ Paramètres } & \multicolumn{3}{|c|}{ Anions (mg.L ${ }^{-1}$ ) } & \multicolumn{4}{|c|}{ Cations (mg.L ${ }^{-1}$ ) } & \multicolumn{4}{|c|}{ Cations en trace $\left(\mu \mathrm{g} . \mathrm{L}^{-1}\right)$} \\
\hline & pH & $\begin{array}{c}\mathbf{T} \\
\left({ }^{\circ} \mathbf{C}\right)\end{array}$ & $\begin{array}{c}\mathbf{C E} \\
\left(\mu \mathrm{S} . \mathrm{cm}^{-1}\right)\end{array}$ & $\begin{array}{c}\text { MTD } \\
\left(\mathrm{mg.L}^{-1}\right)\end{array}$ & $\begin{array}{l}\text { TAC } \\
\left({ }^{\circ} \mathbf{F}\right)\end{array}$ & $\begin{array}{l}\text { THT } \\
\left({ }^{\circ} \mathbf{F}\right)\end{array}$ & $\mathrm{HCO}_{3}^{-}$ & $\mathrm{Cl}^{-}$ & $\mathrm{SO}_{4}{ }^{2-}$ & $\mathrm{NO}_{3}{ }^{-}$ & $\mathrm{Ca}^{2+}$ & $\mathrm{Mg}^{2+}$ & $\mathrm{Na}^{+}$ & $\mathbf{K}^{+}$ & $\mathrm{Al}^{3+}$ & $\mathrm{Fe}^{2+}$ & $\mathrm{Mn}^{2+}$ \\
\hline Minimum (m) & 5,9 & 25 & 61 & 82 & 1,4 & 2,3 & 16,8 & 3,2 & 6 & $<1$ & 5,7 & 2 & 3,6 & 3,8 & $<5$ & $<5$ & $<5$ \\
\hline Moyenne $(\mu)$ & 7,1 & 27,1 & 313,8 & 297,9 & 10,8 & 13,5 & 130,6 & 18,2 & 52,2 & 14,9 & 30,9 & 13,8 & 25,8 & 11,3 & 30 & 200 & 40 \\
\hline $\operatorname{Maximum}(\mathrm{M})$ & 7,6 & 30,6 & 1128 & 1054 & 34,7 & 60,4 & 423,2 & 67 & 401,7 & 99,3 & 169,4 & 86,1 & 86,4 & 30,1 & 70 & 1400 & 300 \\
\hline Ecart Type $(\sigma)$ & 0,4 & 1 & 212,6 & 172,8 & 7,3 & 11,4 & 87,8 & 13,7 & 74,7 & 22,4 & 28,3 & 15,7 & 13 & 5,9 & 20 & 300 & 60 \\
\hline $\mathrm{Cv}=(\sigma / \mu)$ & 0,05 & 0,04 & 0,68 & 0,58 & 0,67 & 0,84 & 0,67 & 0,75 & 1,43 & 1,5 & 0,91 & 1,14 & 0,5 & 0,52 & 1,54 & 1,48 & 0,67 \\
\hline
\end{tabular}

Tableau 2: Teneur en ions $\mathrm{Fe}^{2+}, \mathrm{Mn}^{2+}$ et $\mathrm{Al}^{3+}$ dans quelques eaux de socle.

\begin{tabular}{|c|c|c|c|c|c|c|c|c|c|c|c|c|}
\hline \multirow{2}{*}{ Variables } & \multicolumn{3}{|c|}{ Yao (2009) } & \multicolumn{3}{|c|}{ Goné et al. (2004) } & \multicolumn{3}{|c|}{ Kortatsi et al. (2007) } & \multicolumn{3}{|c|}{ Lasm et al. (2008) } \\
\hline & Min & $\operatorname{Max}$ & Moy & Min & Max & Moy & Min & Max & Moy & Min & $\operatorname{Max}$ & Moy \\
\hline $\mathrm{Fe}^{2+}$ & $<0,005$ & 1,40 & 0,20 & 0,02 & 1,32 & 0,40 & 0,003 & 16,8 & 1,276 & 0,00 & 12,00 & 0,70 \\
\hline $\mathrm{Mn}^{2+}$ & $<0,005$ & 0,30 & 0,04 & 0,00 & 0,25 & 0,40 & 1,00 & 1020 & 117 & 0,00 & 0,86 & 0,10 \\
\hline $\mathrm{Al}^{3+}$ & $<0,005$ & 0,07 & 0,03 & nd. & nd. & nd. & 0,02 & 2,39 & 0,1 & nd. & nd. & nd. \\
\hline
\end{tabular}


T.K. YAO et al. / Int. J. Biol. Chem. Sci. 6(6): 7069-7086, 2012

Tableau 3: Gamme de variation des teneurs en ions majeurs de quelques eaux en zone de socle (Côte d'Ivoire).

\begin{tabular}{|c|c|c|c|c|c|c|c|c|c|c|c|c|c|c|c|c|c|c|}
\hline \multirow{2}{*}{ Variables } & \multicolumn{3}{|c|}{ Biémi (1992) } & \multicolumn{3}{|c|}{ Tapsoba (1995) } & \multicolumn{3}{|c|}{ Soro (2002) } & \multicolumn{3}{|c|}{ Goné et al. (2005) } & \multicolumn{3}{|c|}{ Oga et al. (2009) } & \multicolumn{3}{|c|}{ YAO (2009) } \\
\hline & Min & Max & Moy & Min & Max & Moy & Min & Max & Moy & Min & Max & Moy & Min & Max & Moy & Min & Max & Moy \\
\hline $\mathrm{T}^{\circ} \mathrm{C}$ & 21 & 30 & 27,4 & 26 & 32 & 28,2 & 25,6 & 28,8 & 27,3 & 24,5 & 28,6 & Inc. & 25 & 30 & 27,3 & 25,3 & 30,6 & 27,1 \\
\hline $\mathrm{pH}$ & 4,8 & 7,8 & Inc. & 6 & 8,5 & 6,97 & 5,2 & 7,19 & 6,08 & 4,5 & 7,5 & Inc. & 5,9 & 7,72 & 6,6 & 6,2 & 7,7 & 7,1 \\
\hline Cond $\left(\mu \mathrm{S} . \mathrm{Cm}^{-1}\right)$ & 11 & 1750 & Inc. & 280 & 1450 & 528,3 & 47,4 & 1457 & 342 & 12,1 & 372 & Inc. & 113 & 2460 & 792,3 & 61 & 1128 & 313,3 \\
\hline $\mathrm{Na}^{+}$ & 0,5 & 0,96 & Inc. & 0,1 & 5,5 & 1,28 & 0,13 & 8,16 & 1,21 & 0,01 & 1,59 & Inc. & 0,027 & 6,2 & 1,78 & 0,16 & 3,76 & 1,1 \\
\hline $\mathrm{K}^{+}$ & 0,3 & 0,61 & Inc. & 0,1 & 0,8 & 0,08 & 0,1 & 0,32 & 0,13 & 0,02 & 0,33 & Inc. & 0,003 & 0,46 & 0,1 & 0,09 & 0,77 & 0,3 \\
\hline $\mathrm{Ca}^{2+}$ & 2,3 & 2,58 & Inc. & Inc. & Inc. & 1,1 & 0,1 & 7,13 & 1,5 & 0,13 & 13,6 & Inc. & 0,278 & 10,7 & 2,5 & 0,28 & 8,46 & 1,5 \\
\hline $\mathrm{Mg}^{2+}$ & 1,6 & 1,91 & Inc. & Inc. & Inc. & 1,3 & 0,2 & 3,57 & 0,58 & 0,26 & 9,12 & Inc. & 0,263 & 8,15 & 1,99 & 0,17 & 7,08 & 1,1 \\
\hline $\mathrm{Cl}-$ & 0,4 & 0,59 & Inc. & Inc. & 6,01 & 1,59 & 0,28 & 4,29 & 0,47 & 0 & 1 & Inc. & 0,127 & 15,2 & 2,98 & 0,09 & 1,89 & 0,5 \\
\hline $\mathrm{SO}_{4}{ }^{2-}$ & 0,8 & 1,57 & Inc. & 0 & 1,25 & 0,26 & 0 & 2,4 & 0,26 & 0,02 & 0,6 & Inc. & 0,245 & 3,79 & 1,25 & 0,12 & 8,36 & 1,1 \\
\hline $\mathrm{HCO}_{3-}$ & 2 & 3,13 & Inc. & Inc. & INC. & 2,03 & 0,39 & 13,1 & 2,51 & 0,7 & 7,88 & Inc. & 0,55 & 8,7 & 3,78 & 0,27 & 6,94 & 2,1 \\
\hline $\mathrm{NO}_{3-}$ & 0,6 & 0,65 & Inc. & Inc. & INC. & 1,39 & 0 & 0,22 & 0,17 & 0,01 & 0,36 & Inc. & $<0,001$ & 0,16 & 0,1 & $<0,001$ & 0,16 & 0,3 \\
\hline
\end{tabular}

Max : maximum, Min : minimum, Moy: moyenne ; Inc : inconnue ; Cond : conductivité 
T.K. YAO et al. / Int. J. Biol. Chem. Sci. 6(6): 7069-7086, 2012

Tableau 4: Norme de potabilité chimique des ions analysés.

\begin{tabular}{|c|c|c|c|c|c|}
\hline \multirow[t]{2}{*}{ Paramètres $\left(\mathrm{mg.L}^{-1}\right)$} & \multicolumn{2}{|c|}{ Normes usuelles } & \multicolumn{3}{|c|}{$\begin{array}{c}\text { Valeurs dans les eaux souterraines de } \\
\text { Soubré } \\
\end{array}$} \\
\hline & Admis. & Accep. & Moy & Min & Max \\
\hline Cond $(\mu$ S.Cm-1) & 2000 & 400 & 313,3 & 61 & 1128 \\
\hline $\mathrm{T}\left({ }^{\circ} \mathrm{C}\right)$ & 25 & 15 & 27,1 & 25,3 & 30,6 \\
\hline $\mathrm{pH}$ & 9,5 & 15 & 7,1 & 5,9 & 7,7 \\
\hline $\mathrm{Na}^{+}$ & 100 & $<20$ & 25,8 & 3,6 & 86,4 \\
\hline $\mathrm{K}^{+}$ & 12 & $<10$ & 11,3 & 3,8 & 30,1 \\
\hline $\mathrm{Ca}^{2+}$ & 100 & & 30,9 & 5,7 & 169,4 \\
\hline $\mathrm{Mg}^{2+}$ & 50 & 30 & 13,8 & 2 & 86,1 \\
\hline $\mathrm{Cl}^{-}$ & 600 & 250 & 18,2 & 3,2 & 67 \\
\hline $\mathrm{SO}_{4}{ }^{2-}$ & 400 & 200 & 52,2 & 6 & 401,7 \\
\hline $\mathrm{HCO}_{3}^{-}$ & & & 130,6 & 16,8 & 423,2 \\
\hline $\mathrm{NO}_{3}^{-}$ & 50 & 45 & 14,9 & $<1$ & 99 \\
\hline $\mathrm{Fe}^{2+}$ & 0,2 & 0,3 & 0,2 & $<0,005$ & 1,4 \\
\hline $\mathrm{Mn}^{2+}$ & 0,05 & 0,1 & 0,04 & $<0,005$ & 0,3 \\
\hline $\mathrm{Al}^{3+}$ & 0,2 & & 0,03 & $<0,005$ & 0,07 \\
\hline
\end{tabular}


T.K. YAO et al. / Int. J. Biol. Chem. Sci. 6(6): 7069-7086, 2012

Tableau 5: Inventaire des forages présentant des paramètres hors norme OMS.

\begin{tabular}{|c|c|c|}
\hline Paramètres & Forages hors normes OMS & Fréquence $(\%)$ \\
\hline \multicolumn{3}{|l|}{ Cond. $\left(\mu \mathrm{S} . \mathrm{Cm}^{-1}\right)$} \\
\hline $\mathrm{T}\left({ }^{\circ} \mathrm{C}\right)$ & Tous & 100 \\
\hline \multicolumn{3}{|l|}{$\mathrm{pH}$} \\
\hline \multicolumn{3}{|l|}{$\mathrm{Na}^{+}$} \\
\hline & L13; M11; L12; M1; L8; L4; L41; L11; L11'; M12; L4; L41; L11; & \\
\hline $\mathrm{K}^{+}$ & L11'; M12; L43; L36; M2; L18; L54; L38; M8; L46, & 43,4 \\
\hline $\mathrm{Ca}^{2+}$ & M15 & 1,9 \\
\hline $\mathrm{Mg}^{2+}$ & L8; L39 & 3,8 \\
\hline $\mathrm{Cl}^{-}$ & & 0 \\
\hline $\mathrm{SO}_{4}{ }^{2-}$ & M15 & 1,9 \\
\hline $\mathrm{HCO}^{-}$ & & 0 \\
\hline $\mathrm{NO}_{3-}^{-}$ & L13; L23; L11; L2; SAO; L14, L28 & 13,2 \\
\hline $\mathrm{Fe}^{2+}$ & L32; L31; L8; L2; L39; L48; L36; L24; L6; K2; L44; L46; L18 & 24,5 \\
\hline $\mathrm{Mn}^{2+}$ & L32; L23; L8; L11'; L39, L48; L28; L24; M9; L16; L18; L14; L19 & 24,5 \\
\hline
\end{tabular}


T.K. YAO et al. / Int. J. Biol. Chem. Sci. 6(6): 7069-7086, 2012

Tableau 6: Comparaison des certaines eaux de Soubré avec des eaux minérales de commerce.

\begin{tabular}{|c|c|c|c|c|c|c|c|c|c|c|c|c|c|c|}
\hline $\mathbf{N}^{\circ}$ & EAUX & Cond $(\mu \mathrm{s} / \mathrm{cm})$ & pH & THT $\left({ }^{\circ} \mathbf{F}\right)$ & SIO2 & $\mathbf{C a}$ & Mg & $\mathbf{N a}$ & $\mathbf{K}$ & $\mathrm{HCO}_{3}$ & $\mathrm{CO}_{3}$ & Cl & $\mathrm{SO}_{4}$ & $\mathrm{NO}_{3}$ \\
\hline 1 & + Awa (Côte d'Ivoire) & 395 & 8 & 16 & 50 & 67 & 3 & 21 & 4 & 259 & 0 & 9 & 7 & 0 \\
\hline 2 & + Volvic (France) & 150 & 7 & 4 & 30 & 9 & 6 & 9 & 5 & 61 & 0 & 6 & 6 & 5 \\
\hline 3 & + Evian (France) & 500 & 7 & 30 & 14 & 78 & 24 & 6 & 1 & 357 & 0 & 2 & 10 & 4 \\
\hline 4 & + Sidi Ali (Maroc) & & & & & 22 & 7 & 27 & 3,4 & 98 & 0 & 19 & 32 & 5 \\
\hline 5 & * M16 (Soubré) & 1128 & 7,5 & 60 & & 169 & 44 & 36 & 10 & 369 & 0 & 19 & 402 & $<1$ \\
\hline 6 & *L8 (Soubré) & 643 & 6,55 & 40 & & 33 & 78 & 30 & 30 & 395 & 0 & 15 & 121 & $<1$ \\
\hline 7 & *L39 (Soubré) & 971 & 7,18 & 47 & & 47 & 86 & 52 & 7 & 170 & 0 & 6 & 391 & $<1$ \\
\hline
\end{tabular}

Les teneurs des ions sont en $\mathrm{mg} \mathrm{L}^{-1} ;(+)=$ Eaux minérales de commerce ; $(*)=$ Eaux spéciales de Soubré 


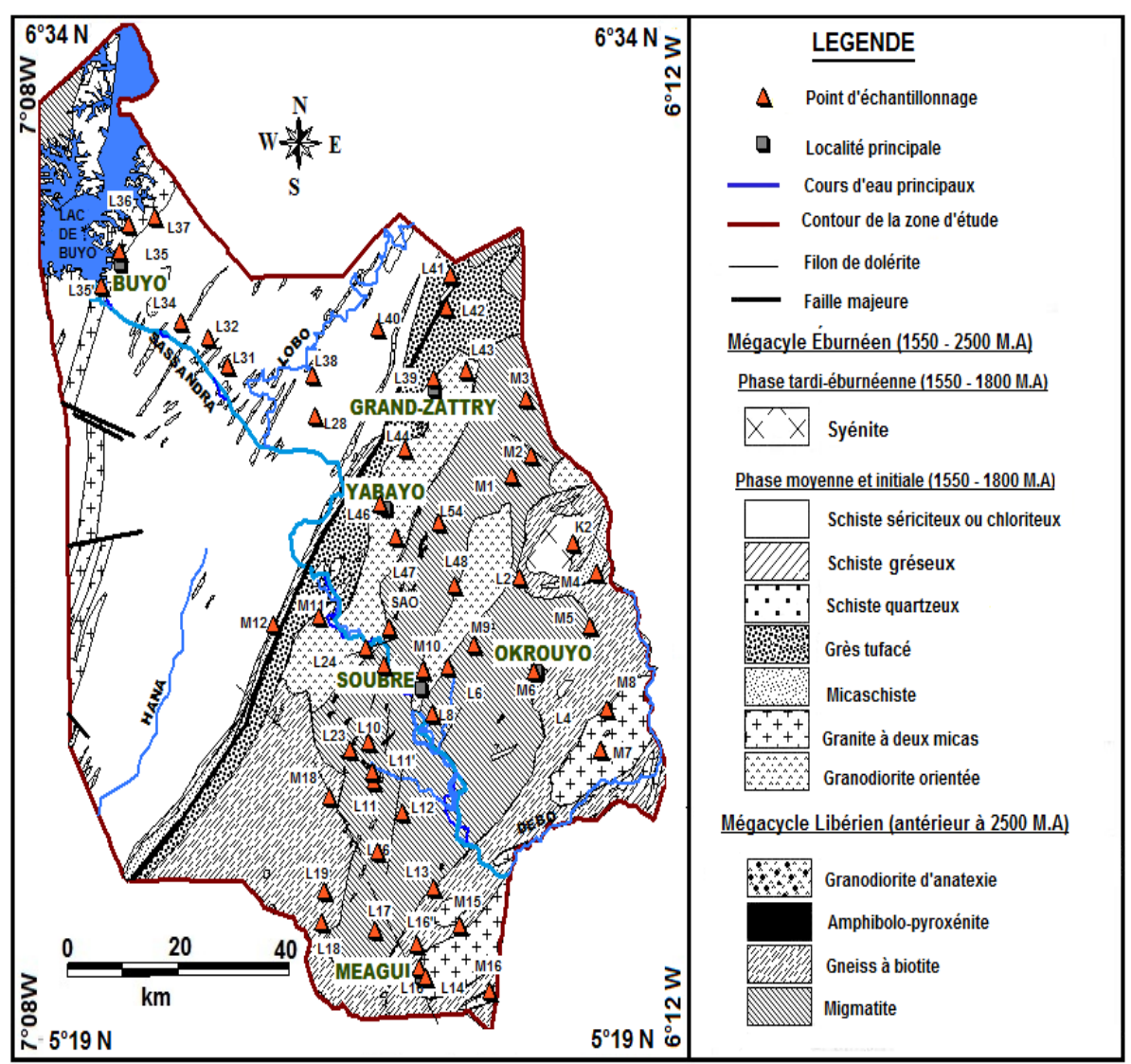

Figure 2: Carte géologique simplifiée d'après Papon et Lemarchand (1973) et point d'échantillonnage d'eau.

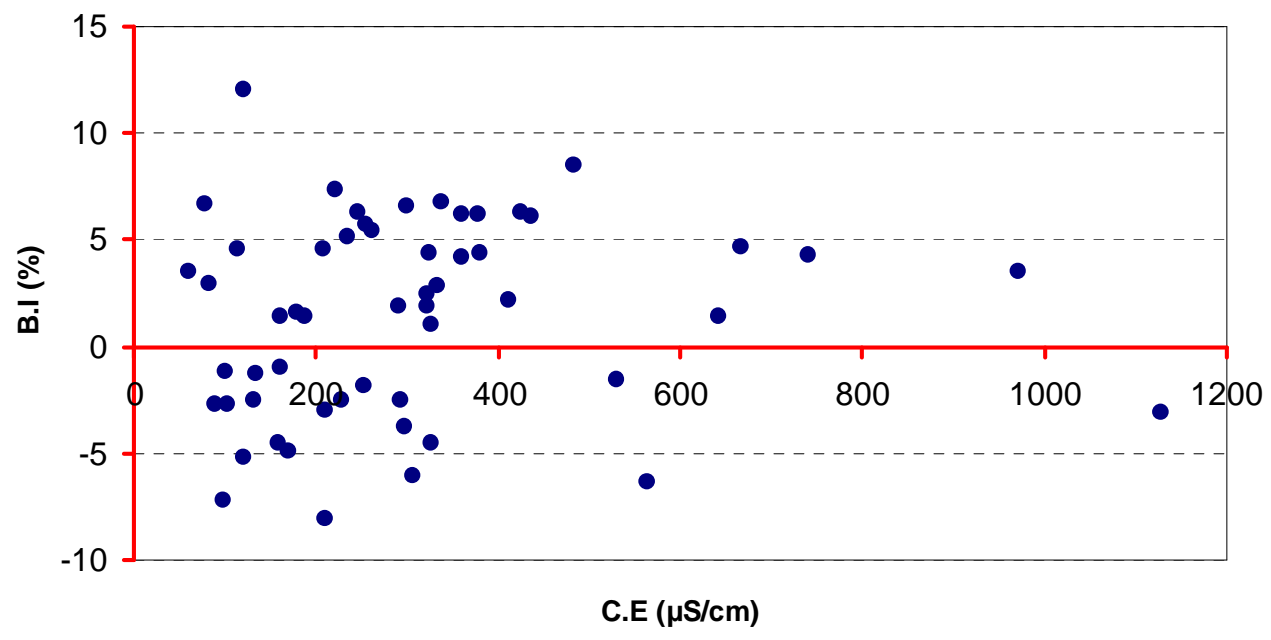

Figure 3: Relation entre la balance ionique (BI) et la conductivité électrique (CE). 


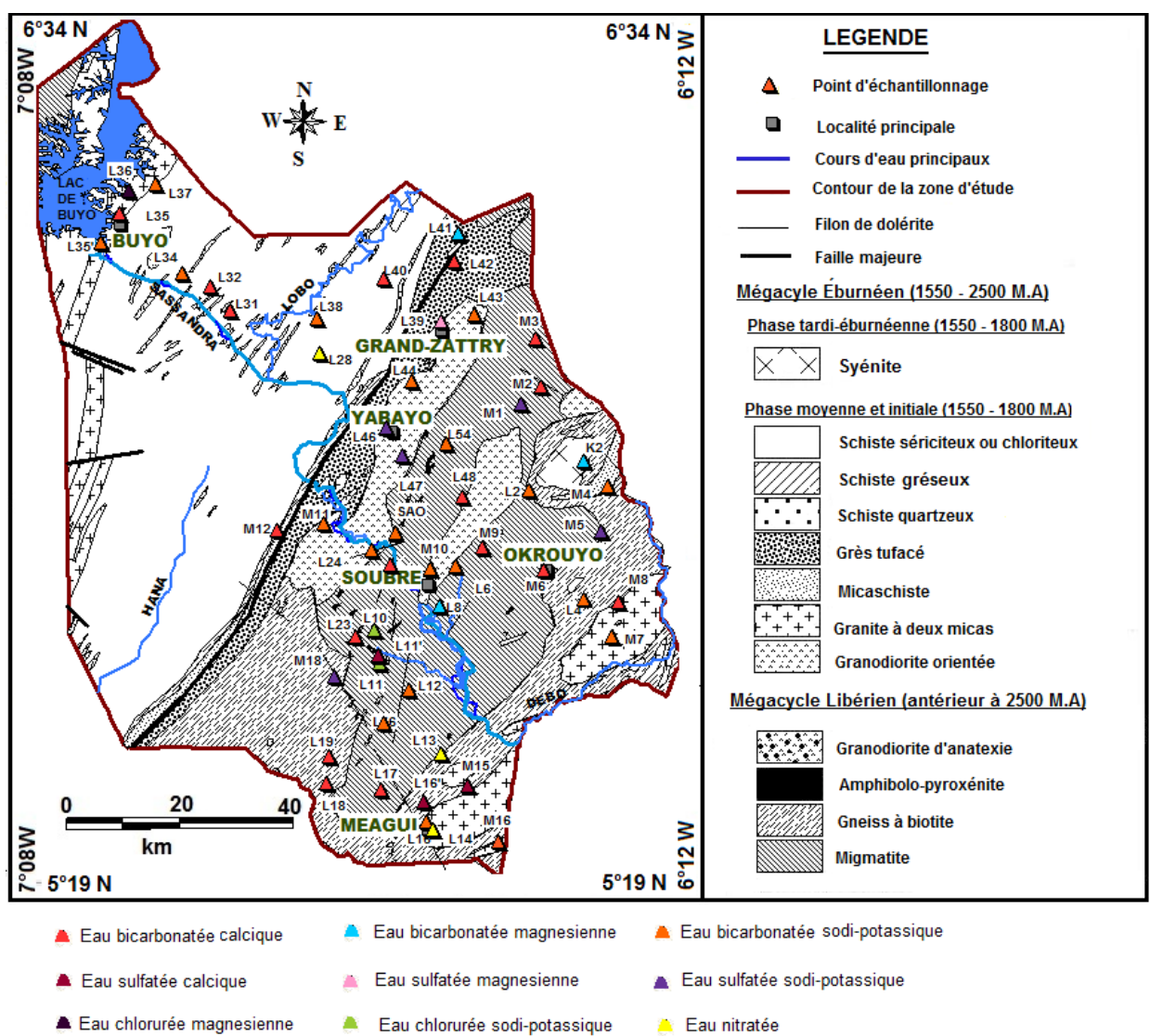

Figure 4: Carte de distribution spatiale des faciès hydrochimiques.

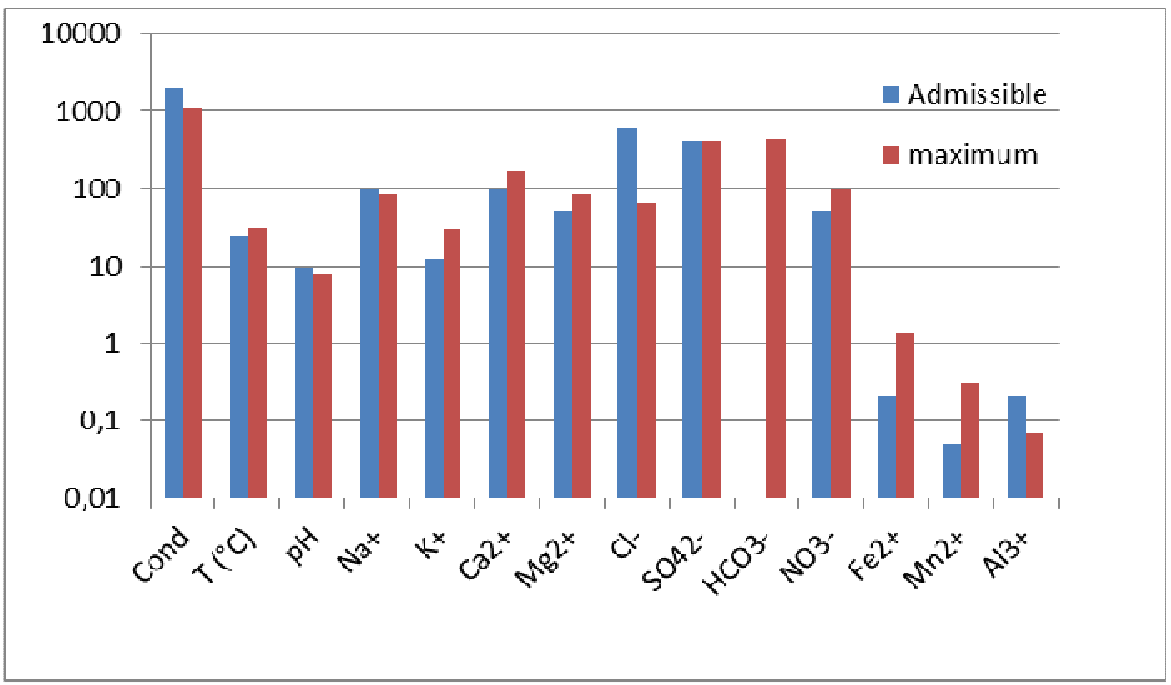

Figure 5: Histogramme de comparaison des teneurs maximales des eaux de Soubré et les teneurs admissibles (OMS, 2008). 


\section{DISCUSSION}

La comparaison de nos résultats avec ceux de plusieurs travaux antérieurs a permis d'aboutir à plusieurs constats. La moyenne de température des eaux de Soubré $\left(27^{\circ} \mathrm{C}\right)$ est similaire à celle des eaux de socle en Côte d'Ivoire (Biémi, 1992 ; Tapsoba, 1995 ; Goné, 2001; Soro 2002 ; Yao, 2005, Oga et al., 2009). Quant aux valeurs de pH, elles sont caractéristiques des eaux de socle c'est-à-dire moins acides que les eaux du bassin sédimentaire de Côte d'Ivoire. Les valeurs de la conductivité électrique sont sensiblement identiques à celles de Soro (2002). En revanche, elles diffèrent totalement des autres auteurs. La proximité de la zone d'étude de celle de cet auteur serait une explication plausible. S'agissant de Goné et al. (2005), ils ont échantillonné des eaux faiblement minéralisées dans l'Ouest de la Côte d'Ivoire où les formations encaissantes présentent un caractère généralement acide. Quant aux paramètres chimiques, les teneurs en fer dans les eaux de Soubré sont inférieures à celles observées par Lasm et al. (2008) et Kortatsi et al. (2007) qui ont travaillé respectivement dans le sud de la Côte d'Ivoire et du Ghana dans des formations géologiques similaires. En revanche, nos résultats montrent des valeurs supérieures à celles observées par Goné et al. (2004) dans les eaux du sud-est de la Côte d'Ivoire. La relative richesse en fer de certaines eaux souterraines est un témoin de la désoxygénation de l'eau par l'activité organique dans le sol et dans la zone insaturée (Michard, 2002). Elle est donc liée au degré d'altération de la roche, au temps de séjour et potentiel d'oxydoréduction. L'eau de la rivière Lobo, affluent du fleuve Sassandra présente une teneur relativement importante en fer $\left(1,1 \mathrm{mg} \cdot \mathrm{L}^{-1}\right)$. Cette concentration en fer proviendrait des horizons supérieurs du profil d'altération où le fer présent n'a pas été remobilisé par les eaux d'infiltration (Lasm et al., 2008) mais a été entraîné par l'eau de ruissellement vers ce cours d'eau. Concernant les ions majeurs, les eaux étudiées présentent des gammes de variations qui sont quasi identiques à celles de Soro (2002) et proches de celles de Biémi (1992), Tapsoba (1995), Oga et al. (2009). Elles sont différentes de celles de Goné et al. (2005). Cela semble logique vu les raisons avancées plus haut. Aussi, constate-t-on que le faciès des eaux souterraines n'est pas lié à une formation géologique. Les deux faciès les plus représentés, les eaux bicarbonatées calciques et les eaux bicarbonatées sodi-potassiques sont observées dans toutes les formations géologiques. Ce constat est valable aussi pour d'autres faciès. Réciproquement, en considérant une lithologie particulière, par exemple dans les schistes de l'unité HanaLobo, on retrouve ces deux faciès. Cependant, les eaux des grès tuffacés sont seulement bicarbonatées calco-magnésiennes. Ces types d'eaux peuvent subir des mélanges ou être influencés par les apports extérieurs. Le mélange se fait à la suite de l'infiltration (renouvellement) et l'intercommunication entre aquifères se fait par le biais des zones de faiblesse (fractures ouvertes, couloirs de cisaillement, filons altérés) et le dense réseau de petites fractures qui sont éparses dans le département (Yao et al., 2012). Ces différents échanges entre certains aquifères sont responsables de l'hétérogénéité dans la distribution spatiale des faciès hydrochimiques. Ce constat est en accord avec Tonetto et Bonotto (2005) et Yao (2005 et 2009), qui affirment que dans les terrains de socle, la qualité chimique de l'eau souterraine tend à être fortement variable d'un endroit à un autre et parfois d'une saison à l'autre. En définitive, nos résultats montrent que les eaux de Soubré sont chimiquement quasi similaires à celles du socle cristallin et cristallophyllien de la Côte d'Ivoire. En revanche, les eaux de Soubré présentent des particularités qui méritent d'être détaillées à la lumière des directives de l'Organisation Mondiale de la Santé (OMS).

\section{Spécificité chimique des eaux souterraines de Soubré}

La première spécificité est relative aux paramètres qui ne posent aucun problème de potabilité. Les valeurs observées sont dans les limites préconisées par l'OMS. Il s'agit de la conductivité des eaux, le $\mathrm{pH}$, le sodium, le chlorure et l'aluminium. La seconde observation concerne la température de l'eau 
souterraine de Soubré. Comparée à la directive de l'OMS, l'eau de Soubré semble chaude. Mais, cette région se trouvant dans un pays tropical, la température ambiante moyenne descend très rarement en dessous de $25{ }^{\circ} \mathrm{C}$. C'est pourquoi, selon Rodier (1996), $30{ }^{\circ} \mathrm{C}$ doit être considérée comme une température moyenne des eaux souterraines en Afrique de l'Ouest à cause des conditions climatiques. Les eaux «spéciales » qui sont riches en fer, manganèse, magnésium, potassium et en sulfates mettent en évidence que certaines eaux souterraines de la région circulent dans des fractures profondes d'origine tectonique. L'encaissant serait encore sain (non altéré) donc, les eaux hydrolysent les minéraux ferromagnésiens tels que les micas (Grimaldi et al., 2004). En plus, ces eaux ne montrent pas de signes de pollution récente vue, les faibles teneurs de nitrate (Faillat, 1990). Aussi, note-t-on que les « eaux spéciales » ont des teneurs en certains ions aussi intéressantes que des eaux minérales. Mais souvent, à cause du goût désagréable dû au calcium et / ou sulfate, elles ne sont pas souvent consommée par la population. Enfin, il faut signaler des teneurs élevées en nitrate dans plusieurs échantillons analysés. Le fait d'avoir des taux importants en nitrate dans les eaux souterraines est un indice de la vulnérabilité de ces eaux souterraines car elles sont considérées comme étant à l'abri de la pollution (Nkotagu, 1996, Jaouad et al., 2007). Quel est donc l'origine de cet ion?

Selon Faillat (1990), plusieurs sources de nitrate ont été établies. Il s'agit des roches, des apports météoriques, des engrais, de la végétation décomposée des sols, et enfin des polluants domestiques. Cependant, dans le cas de la zone d'études deux sources principales peuvent être envisagées venant principalement des activités anthropiques. Primo, l'apport de nitrate par les polluants domestiques semble être la principale source d'enrichissement en nitrate des eaux souterraines. En effet, la majorité des forages échantillonnés se trouvent aux abords immédiats des villages ou même au milieu des habitations où l'insalubrité est remarquable. Nous avons constaté la présence de broussailles, d'ordures, d'eaux stagnantes et verdâtres, etc. En outre, le vieillissement de certains ouvrages amène à penser que les équipements installés pour les protéger contre la pollution d'origine anthropique ne sont plus efficaces. Par conséquent, les eaux de forages deviennent vulnérables aux polluants organiques issus des activités domestiques: latrines, dépôts d'ordures, infiltration des eaux sales, etc. (Traoré et al., 2006). Elles sont d'autant plus vulnérables que plusieurs forages sont situés dans des zones basses de la topographie locale. Cela souligne l'intérêt primordial de favoriser l'implantation de fosses septiques dans les villages et campements. Secundo, la mise en culture des zones forestières après défrichage et brûlis préalable constatée dans la région est source de production de nitrate. La destruction de la forêt produit de la matière organique qui, en se décomposant libère de l'azote qui sera lessivé à la suite des pluies abondantes qui tombent sur la région. Ce lessivage entraine la pollution des eaux souterraine, car l'infiltration transfert naturellement le nitrate vers les nappes souterraines (Nkotagu, 1996). L'azote représente en général, environ 3,5\% en poids d'une plante vivante et $1,5 \%$ de sa matière sèche. Or en forêt intertropicale humide, la matière végétale peut représenter jusqu'à 1500 tonnes à l'hectare (Soro, 2002). Dans les sols, sur $50 \mathrm{~cm}$ d'épaisseur, le stock est de l'ordre de 2 à 7 tonnes par hectare dans le sud de la Côte d'Ivoire (Deirdre et Birmingham, 2003). Dans un environnement qui devient réducteur, après la consommation de l'oxygène, le $\mathrm{NO}_{3}{ }^{-}$est le plus fort accepteur d'électrons qui soit capable d'oxyder le carbone organique. Le processus de dénitrification exige un (ou des) donneur(s) d'électrons pour rendre possible la réduction de $\mathrm{NO}_{3}{ }^{-}$en $\mathrm{N}_{2}$ gaz, les plus courants étant le carbone organique et le fer réduit puis les espèces soufrées réduites (Faillat et al., 1999). La pollution des eaux par les nitrates présente un double risque. Ingérés en trop grande quantité, les nitrates ont des effets toxiques sur la santé humaine. Par ailleurs, ils contribuent avec les phosphates à modifier l'équilibre biologique des milieux aquatiques en provoquant des phénomènes 
d'eutrophisation (Ayraud, 2005). La présence de nitrate dans les eaux souterraines de la région (pollution d'origine antropique) montre que les nappes sont vulnérables aux produits phytosanitaires notamment le glyphosate (issu du Roundup). Cet herbicide abondamment utilisé par les paysans (Yao, 2009), serait lessivé dans les sols, et se retrouve dans les rivières et les eaux souterraines. Ceci est d'autant possible car au Danemark, en 2003, le glyphosate a été retrouvé à une concentration cinq fois supérieure à la norme, dans l'eau potable (Borvon, 2006). Cette molécule est également présente en grande quantité dans les eaux de certaines régions françaises (Miguel, 2003). Les sociétés de distribution d'eau potable font payer aux consommateurs, le surcoût engendré pour le traitement de ce produit. À Soubré, lors de nos missions de terrain, nous avons constaté que les pompes de nombreuses localités étaient hors service ou il n'existe carrément pas de points d'eau potable. Par conséquent, les habitants ont recours aux eaux de surface (rivières, marigots et mares) et de puits peu profonds ( $4 \mathrm{~m}$ en moyenne) pour leurs besoins en eau. Il serait donc fort probable que ces populations ingèrent des molécules issues de ces produits. Même, celles qui ont la chance d'avoir un accès à l'eau souterraine (considérée comme potable), ne sont pas à l'abri de l'ingestion des dérivés des produits phytosanitaires. Le manque d'information et de travaux sur les conséquences de l'impact éventuel des produits phytosanitaires sur les eaux souterraines de Soubré, nous a conduit à voir dans la littérature pour montrer les conséquences de ces produits sur la santé de la population. L'agence américaine de protection de l'environnement (US Environmental Protection Agency) détaille les effets nocifs sur la santé que pourrait provoquer l'exposition à de fortes doses. Ce sont : la congestion des poumons, l'accélération du rythme de la respiration à court terme, « l'endommagement des reins », les effets sur la reproduction à long terme (Goldstein et al., 2002). Benachour et al. (2008) ont mis en évidence l'impact de diverses formulations et constituants de ce pesticide sur des lignées cellulaires humaines (cellules néonatales, cellules placentaires et de rein d'embryon). Ils ont mentionné aussi diverses atteintes (nécrose, asphyxie, dégradation de l'ADN, etc.) induites soit par le glyphosate, soit par l'AMPA ou encore le POEA.

\section{Conclusion}

Cette étude a consisté à l'évaluation chimique des ressources en eau de département de Soubré, zone très importante de production de cacao. Pour y arriver, les paramètres physico-chimiques ont été utilisés pour définir le critère de potabilité. Les eaux souterraines des aquifères discontinus de Soubré sont en général moyennement minéralisées avec des $\mathrm{pH}$ autour de 7. Elles sont à majorité de type bicarbonaté et certaines présentent d'importantes teneurs de nitrates, fer, manganèse et en sulfate. Les eaux souterraines bien qu'elles soient semblables aux eaux de socle cristallin et métamorphique de régions tropicales, elles présentent par endroits des minéralisations qui les rapprochent des eaux minérales. Aussi, observe-t-on des teneurs très critiques en sulfate, en nitrate et en fer dans plusieurs échantillons. Le nitrate, éléments dangereux à de fortes teneurs, est un très bon indicateur de la vulnérabilité des aquifères. Les fortes teneurs de nitrate constatées permettent de soupçonner la présence de dérivées des produits phytosanitaires et pesticides dans les eaux de consommation. Ces produits à l'exemple du Roundup entrainent une toxicité chez le consommateur d'où l'importance de mener à court terme une étude complète de leurs concentrations dans tout le sud-ouest ivoirien afin de cibler les localités exposées à un risque sanitaire. À long terme, nous comptons conduire une étude de la qualité biologique des points d'approvisionnement en eau villageois (puits, marigots, rivières et forages) dans toute la nouvelle boucle de cacao (Sud-Ouest de la Côte d'Ivoire).

\section{RÉFÉRENCES}

AFNOR (Association française de normalisation). 1997. Qualité de l'Eau : Terminologie, Echantillonnage et Evaluation des Méthodes (Tome 1, 2e édn). AFNOR : Paris, France ; 376 p. 
Ayraud V. 2005. Détermination du temps de résidence des eaux souterraines : application au transfert d'azote dans les aquifères fracturés hétérogènes. Thèse de l'Université de rennes 1, p. 298.

Barcelona M, Gibb J, Helfrich JA, Garske EE. 1985. Practical guide for groundwater sampling. Illinois State Water survey report, p. 374.

Benachour N, Seralini GE. 2008. Gyphosate formulations induce apoptosis and necrosis in human umbilical embryonic and placental cells. Chem. Res. Toxicol., 22: $97-105$.

Biémi J. 1992. Contribution à l'étude géologique hydrogéologique et par télédétection des bassins versants subsahariens du socle précambrien d'Afrique de l'ouest : hydrostructurale, hydrodynamique, hydrochimie et isotopique des aquifères discontinus de sillons et aires granitiques de la haut Marahoué (Côte d'Ivoire). Thèse de Doctorat d'État, Université d'Abidjan, p. 480.

Bourvon G. 2006. Pollution de l'eau : du glyphosate dans les nappes. http://seaus.free.fr/spip.php?article27. Consulté en aout 2012

Deirdre M, Birmingham. 2003. Local knowledge of soils: the case of contrast in Côte d'Ivoire. Geoderma, 111: 481-502.

Faillat J-P. 1990. Origine des nitrates dans les nappes de fissures de la zone tropicale humide: exemple de la Côte d'Ivoire. Journal of Hydrology, 113(1-4): 231-264.

Faillat J-P, Somlette L, Sicard T. 1999. Contrôles redox et hydrodynamiques des nitrates et zonation hydrochimique verticale des nappes de fissures du socle. Possibilité de généralisation. Bulletin de la Société Geologique de France, 170(2): 229-240.

Franz JE, Mao MK, Sikorski JA. 1997. Glyphosate: a unique global herbicide. ACS Monograph 189. Am. Chem. Soc., 163-175.

Goldstein DA, Acquavella JF, Mannion RM, Farmer DR. 2002. An analysis of glyphosate data from the California Environmental Protection Agency pesticide illness surveillance program. $J$. Toxicol. Clin. Toxicol., 40: 885-892.

Goné D. 2001. Contribution de l'étude des paramètres physico-chimiques des eaux souterraines à la compréhension du fonctionnement des systèmes hydrauliques en milieu fissurés de la région semi montagneuse de Man (Ouest de la Côte d'Ivoire). Thèse de Doctorat, Université d'Abobo-Adjamé, p.182.

Goné D, Savané I, Goblé M. 2004. Caractéristiques physico-chimiques majeures des eaux souterraines des aquifères de fissures de la région d'Agboville (Sud-Ouest de la Côte d'Ivoire). Rev. Ivoir. Sci.Technol., 5: 117133.

Goné D, Savané I, N'go YA, Biémi J. 2005. Mobilité relative des cations lors de l'altération des roches et acquisition de la minéralisation des eaux souterraines dans le degré carré de Man (Ouest de la Côte d'Ivoire). Sciences de la Nature, 2: 85-94.

Grimaldi C, Viaud V. 2004. "Stream nitrate variation explained by ground water head fluctuation in a pyrite-bearing aquifer." Journal of Environmental Quality, 33: 994-1001.

Hem J. 1989. Study and interpretation of the chemical characteristics of natural waters. US Geological Survey Water Supply paper, 2254, p. 264.

Jain P, Sharma JD, Sohu D, Sharma P. 2005. «Chemical analysis of drinking water of villages of Sanganer Tehsil, Jaipur District ». Int. J. Environ. Sci. Tech., 2(4): 373-379.

Jaouad EA, Sanae K, Namira EA, Abderrauf H. 2007. Impact des activités anthropiques sur la qualité des eaux souterraines de la communauté Mzamza (Chaouia, Maroc). Revue des Sciences de l'Eau, 20(3): 309-321.

Kortatsi K, Tay K, Anomu G, Hayford E, Dartey A. 2007. Hydrogeochemical evaluation of groundwater in the lower Offin basin Ghana. Environ Geol., 50: 299-311.

Lasm T, Yao KT, Oga M-S, Kouamé F, Jourda JP, Kouadio E, Baka D. 2008. Analyse des caractéristiques physico- 
chimiques des eaux souterraines en zone de socle protérozoïque de la région de Tiassalé (Sud de la Côte d'Ivoire). European Journal of Scientific Research, 20(3): 526-543.

Margat J. 2008. Exploitations et utilisations des eaux souterraines dans le monde. Programme Hydrologique International (PHI), UNESCO, p. 52. http://imta. janium.net/janium/Documentos/208894.p df. Consulté en aout 2012.

Michard G. 2002. Chimie des eaux naturelles : principes de géochimie des eaux. Publication DL, p.461.

Miquel G. 2003. Rapport de l'OPECST n ${ }^{\circ} 215$ (2002-2003) de M. Gérard MIQUEL, fait au nom de l'Office parlementaire d'évaluation des choix scient. Tech. Déposé le 18 mars 2003.

Nkotagu H. 1996. Origins of high nitrate in groundwater in Tanzania. Journal of African Earth Sciences, 21(4): 471-478.

Oga M-S, Lasm, Yao KT, Soro N, Saley MB, Kouassi D, Gnamba F. 2009. Caractérisation chimique des eaux des aquifères de fracture: cas de la région de Tiassalé en Côte d'Ivoire. European Journal of Scientific Research, 31(1): 7287.

OMS 2008. Guidelines for Drinking-Water Quality. Third edition, incorporating the first and second Addenda, Recommendations, OMS, p. 515.

Papon A, Lemarcharnd R. 1973. Géologie et minéralisation du sud-ouest de la Côte d'Ivoire. Synthèse des travaux de l'opération SASCA (1962-1968). SODEMI-Abidjan, p. 284.

Prasad BG, Narayana TS. 2004. « Subsurface water quality of different sampling stations with some selected parameters at Machilipatnam Town ». Nat. Env. Poll. Tech., 3(1) : 47-50.

Rodier JC. 1996. L'analyse de l'Eau (8 édn). Dunod Tech: Paris, France; p.1384.

Soro N. 2002. Hydrochimie et géochimie isotopique des eaux souterraines du carré de Grand-Lahou et ses environs (SudOuest de la Côte d'Ivoire). Implication hydrologique et hydrogéologique. Thèse de Doctorat d'État ès Sc. Nat., Université de Cocody, p. 272.

Tahoux M. 1993. Économie de plantations et organisation de l'espace du sud-ouest ivoirien. Thèse de Doctorat, Université de Cocody, p. 529.

Thierrin J, steffen P, cornaz S, Vuataz F, Loaser. 2001. Guide pratique de l'échantillonnage des eaux souterraines. Édité par l'Office de l'environnement, des forêts et du Paysage et la Société Hydrogéologique de Suisse, p. 91.

Tonetto E, Bonotto D. 2005. Hydrochemical relationships in groundwater from central Sao Paulo State Brazil. Environ. Geol., 47: 942-955.

Tapsoba AS. 1995. Contribution à l'étude géologique et hydrogéologique de la région de Dabou (Sud-Est de la Côte d'Ivoire). Doctorat $3^{\mathrm{e}}$ cycle, Université de Cocody, p. 211.

Traoré SK, Mamadou K, Dembelé A, Lafrance P, Mazellier P, Houenou P. 2006. Contamination de l'eau souterraine par les pesticides en régions agricoles en Côte d'Ivoire (centre, Sud et Sud-Ouest). Journal Africain des Sciences de l'Environnement, 1: 1-9.

Yao KT. 2005. Origine de l'abondance des ions fer et manganèse dans les eaux de forage du socle de la Côte d'Ivoire : cas de la région de Tiassalé. Mémoire de DEA, Université de Cocody, p. 78.

Yao KT. 2009. Hydrodynamisme de l'eau souterraine dans les aquifères de socle cristallin et cristallophyllien du SudOuest de la Côte d'Ivoire : cas du département de Soubré. Apports de la télédétection, de la géomorphologie et de l'hydrogéochimie. Thèse de Doct. Conservatoire National des Arts et Métiers, Paris, p. 284.

Yao KT, Fouché O, Oga M-S, Assoma TV. 2012. Extraction de linéaments structuraux à partir d'images satellitaires et estimation des biais induits, en milieu de socle précambrien métamorphisé. Revue Télédétection, 10(4): 161-178. 